01,07

\title{
Особенности движения дислокаций в алюминии с учетом рельефа Пайерлса в присутствии ультразвука
}

\author{
(C) М.М. Аракелян \\ Ереванский государственный университет, \\ Ереван, Армения \\ E-mail: marakelyan@ysu.am
}

(Поступила в Редакцию 6 марта 2017 г.

В окончательной редакции 31 марта 2017 г.)

\begin{abstract}
Исследовано влияние ультразвука на движение дислокаций Френкеля-Конторовой в алюминии с учетом рельефа Пайерлса. При преодолении барьера Пайерлса дислокация движется с переменной скоростью. Под действием ультразвука различных частот, сопоставимых со временем перехода дислокации в соседнюю долину, меняется длина свободного пробега дислокаций. Получены зависимости напряжения от деформации для больших и малых скоростей деформаций. В обоих случаях имеет место разупрочнение, однако скорость и характер разупрочнения разные. При резонансной частоте уменьшается сопротивление деформированию, укорачивается стадия упрочнения и удлиняется стадия разупрочнения. На графике зависимости коэффициента упрочнения от координаты имеются три области, различные по характеру. При резонансной частоте коэффициент упрочнения уменьшается.
\end{abstract}

DOI: 10.21883/FTT.2017.11.45064.065

В присутствии ультразвука поведение дислокаций в кристаллах меняется вследствие возникающих акустических напряжений. При этом происходит существенное изменение механических характеристик кристалла, например, уменьшение сопротивления деформированию, изменение стандартных кривых напряжениедеформация, зависимость хода деформирования от скорости деформации и т.д. [1]. Исследование воздействия ультразвука на кристаллы на микроуровне и установление влияния возникающих перестроек на макроскопические свойства представляет практический и теоретический интерес.

В настоящей работе рассмотрено движение дислокаций Френкеля-Конторовой в алюминии с учетом рельефа Пайерлса при комнатных и низких температурах и воздействие ультразвука на динамику дислокаций. В чистых металлах при низких температурах основным препятствием при движении дислокаций являются барьеры Пайерлса. При учете рельефа Пайерлса при перемещении дислокации в кристалле ее конфигурация и упругая энергия испытывают периодические изменения. При этом возникают динамические потери, так как периодические изменения конфигурации дислокации и неравномерность ее движения приводят к излучению дислокацией упругих волн, т.е. к внутреннему трению.

В общем случае анализ явления упрочнения с точки зрения дислокационных представлений является сложной задачей. Поэтому нами использована одномерная модель дислокации Френкеля-Конторовой, которая имеет некоторые преимущества по сравнению с другими моделями. В этой модели используется дискретный подход, энергия Пайерлса уменьшается с увеличением ширины дислокации, что соответствует эксперименту, напряжение Пайерлса по порядку величины соответствует получаемым из эксперимента данным: $\sigma_{\mathrm{P}} \sim 10^{-4} \mu-10^{-2} \mu$, $\mu$ - модуль сдвига кристалла [2].
В [3] был разработан метод периодического импульсного нагружения. Этот метод позволяет исследовать динамические свойства дислокаций и получать более полную информацию о движении дислокаций. При этом в зависимости от частоты внешнего поля меняется время перехода дислокации в соседнюю долину потенциального рельефа. Если время действия нагрузки меньше времени образования двойного перегиба критического размера, то дислокация не успевает перейти в соседнюю долину потенциального рельефа, и оставшиеся двойные перегибы аннигилируют. В противном случае дислокация переходит в соседнюю долину и увеличивается ее средний пробег. В алюминии барьер Пайерлса $\sim 4 \cdot 10^{-15} \mathrm{erg}$ [4]. При частоте $\Omega \sim 10^{12} \mathrm{~Hz}$ длительность импульса напряжения сопоставима со временем перехода дислокации в соседнюю долину.

Математическое моделирование движения дислокации в периодическом поле в рамках принятой модели позволяет получать сведения о характеристиках дислокации. Для описания механизмов внутреннего трения и упрочнения получено неоднородное синус-уравнение Гордона с трением и периодическим внешним упругим напряжением $\sigma(t)=\sigma_{0} \exp i \Omega t$, где $\sigma_{0}$ и $\Omega$ соответственно амплитуда и частота внешнего воздействия .

Пусть ось $x$ направлена вдоль равновесного положения прямолинейной дислокации. Тогда уравнение для смещения атомов от положения равновесия будет иметь вид

$$
\begin{aligned}
m \ddot{y}_{n}=f_{0} \sin \frac{2 \pi y_{n}}{a} & +k\left(y_{n+1}-y_{n}\right)-k\left(y_{n}-y_{n-1}\right) \\
& +F_{\mathrm{tr}}+F_{0} \sin [\Omega t]
\end{aligned}
$$

где $y_{n}$ - смещение $n$-го атома от положения равновесия, $m$ - масса атома, $f_{0}=m\left(v_{0}\right)^{2} a / 2 \pi\left(l_{0}\right)^{2}$, где $l_{0}$ - пара- 
метр, который увеличивается при увеличении жесткости пружины и уменьшении силы со стороны подложки, т. е. уменьшается с увеличением барьера Пайерлса, $v_{0}$ скорость звука, $a$ - постоянная решетки.

В безразмерных единицах $\tilde{x}=x \omega / v_{0}, \tilde{t}=t \omega$ уравнение (1) принимает вид неоднородного синус-уравнения Гордона

$$
\ddot{\varphi}_{n}+\sin \varphi_{n}-\varphi_{n}^{\prime \prime}+\beta \dot{\varphi}_{n}=\gamma \sin \frac{\Omega_{t}}{\omega},
$$

где $\omega^{2}=2 \pi f_{0} / m a, \quad v_{0}=a \sqrt{k / m}, \quad \beta=\mu_{0} / m \omega$, $\gamma=2 \pi F_{0} / m a \omega^{2}, \mu_{0}-$ коэффициент, характеризующий трение, $\varphi_{n}$ - смещение $n$-го атома от положения равновесия. При переходе от (1) к (2) предполагается, что размер дислокации много больше межатомного расстояния, что аналогично предположению, что упругая энергия взаимодействия между соседними атомами много меньше энергии взаимодействия с потенциалом подложки.

Исследуем (2) в численном виде. Моделирование осуществлялось с помощью программного пакета Mathematica. Начальные условия выбираем, используя известное аналитическое решение однородного синусуравнения Гордона. При выборе граничных условий считаем, что образец, по которому распространяется дислокация, считается открытым на обоих концах, т. е. $\partial \varphi /\left.\partial x\right|_{x=0}=\partial \varphi /\left.\partial x\right|_{x=l}=0$. При внешней частоте $\Omega \sim 10^{12} \mathrm{~Hz}$ масштаб времени $2.5 \cdot 10^{-6}$.

Рассмотрим зависимость длины свободного пробега дислокации от частоты внешнего упругого поля с учетом силы трения. Из численного эксперимента следует, что дислокации, исходящие из одной точки, со временем останавливаются, причем длина свободного пробега дислокаций разная в зависимости от частоты внешнего переменного поля.

Зависимость длины свободного пробега дислокации от частоты упругого поля при пропускании через кристалл высокочастотного звука $\sim 10^{12} \mathrm{~Hz}$ с коэффициентами $0.06,0.08,0.1,0.25,0.5,1,1.5,2,2.5$ соответственно представлена на рис. 1 .

Из рис. 1 видно, что при определенной частоте длина свободного пробега дислокации максимальна. Очевидно, этой частоте соответствует образование несхлопывающегося двойного перегиба, в результате чего в течение времени действия положительной части внешнего упругого поля дислокация переходит в следующую долину потенциального рельефа [3].

Зависимость напряжения от деформации в области пластичности в макромасштабе принимается, согласно [5], в следующем виде:

$$
\sigma=k \varepsilon^{n}, \quad n=\ln (1+\delta), \quad k=\sigma_{b} e^{n} n^{-n},
$$

где $\sigma-$ напряжение, $\varepsilon-$ накопленная деформация, $\sigma_{b}-$ предел прочности (для алюминия $\left.\sigma_{b}=73.5 \cdot 10^{7} \mathrm{dyn} / \mathrm{cm}^{2}\right)$.

Движение дислокации при учете потенциального рельефа кристалла осуществляется следующим образом. Под действием термической активации происходит обра-

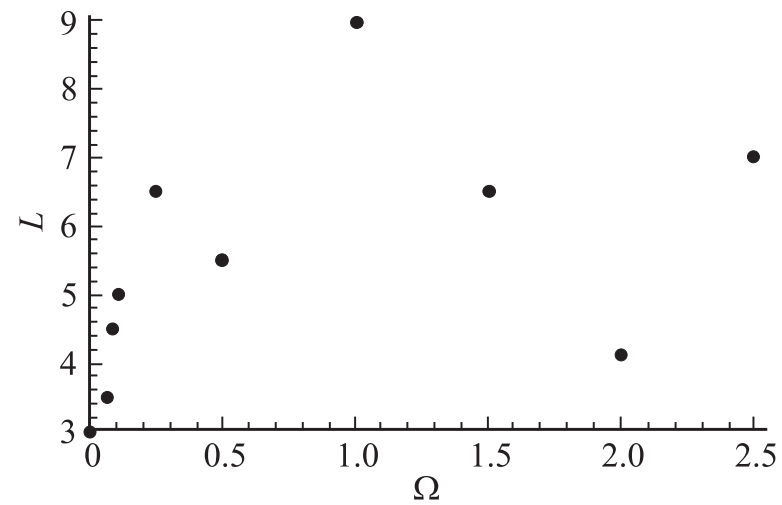

Рис. 1. Зависимость длины свободного пробега дислокаций от частоты переменного упругого поля.

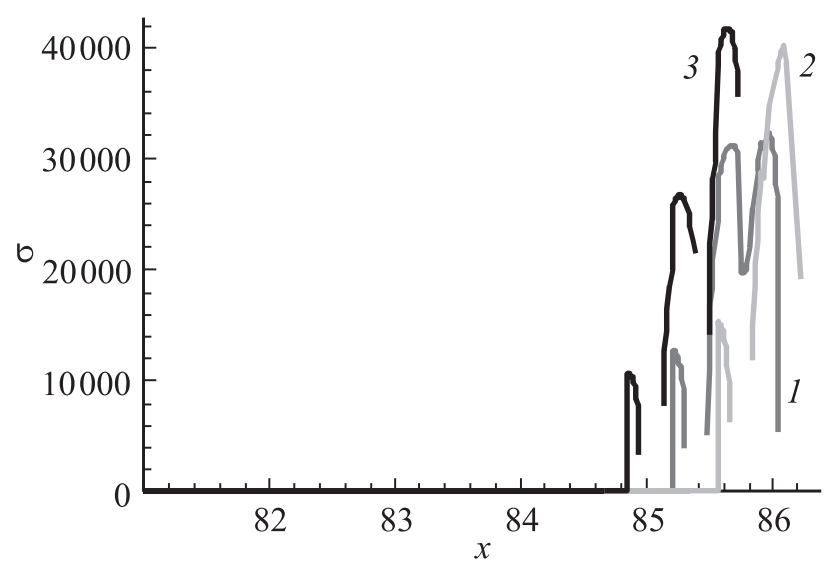

Рис. 2. Зависимости напряжения от координаты для резонансной частоты -1 , близлежащих частот $-2,3$.

зование двойного перегиба на дислокации, дальнейшее движение перегибов в разные стороны происходит под действием очень малых напряжений. Таким образом, акт скольжения осуществляется поэтапно.

Моделированы сравнительные зависимости напряжения от координаты для резонансной частоты и близлежащих частот (для наглядности графики даны для чуть смещенных моментов времени) (рис. 2). Из рис. 2 следует, что барьер Пайерлса существенно меньше для резонансной частоты по сравнению с нерезонансными частотами, что соответствует максимальному значению длины свободного пробега дислокации (рис. 1).

В ГЦК-кристаллах (к ним относится также алюминий) различают 3 участка, на которых действуют различные механизмы деформации и упрочнения [6]. Нами получена зависимость коэффициента упрочнения от координаты без внешнего поля и с внешним полем (рис. 3). Как видно из рис. 3, просматриваются 3 области, причем каждому участку соответствует различная зависимость коэффициента упрочнения от координаты. Наличие резонансной частоты уменьшает коэффициент упрочнения.

Для доказательства того, что вблизи дислокации образуется область микропластичности, построена зави- 


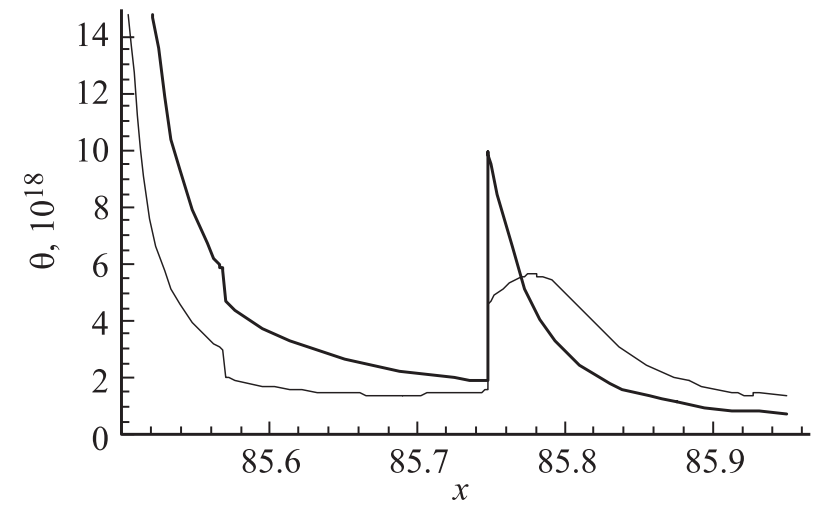

Рис. 3. Зависимость коэффициента упрочнения от координаты в окрестности дислокации (тонкая кривая соответствует случаю, когда прикладывается резонансная частота, жирная кривая - без частоты).

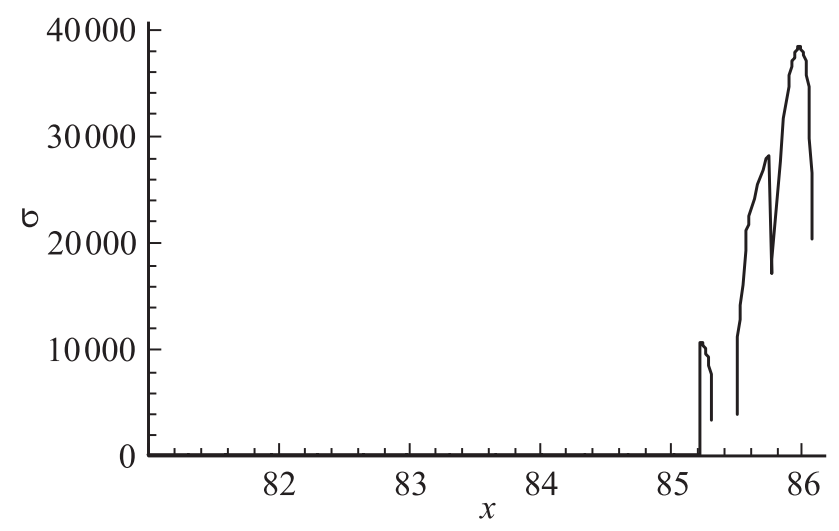

Pис. 4. Зависимость напряжения от координаты в окрестности дислокации.

симость $\sigma(x)$ в окрестности дислокации без внешнего упругого поля (рис. 4).

Как видно из рисунка, максимальное напряжение реализуется вблизи линии дислокации и падает при удалении от нее. Вероятно, осцилляции в этом случае обусловлены периодическим потенциалом Пайерлса.

Исследуем график (рис. 4) для разных интервалов изменения координаты. Если в уравнении (2), написанном без внешней частоты и силы трения, брать $x$ от 85.9 до 85.905 (область в окрестности вершины барьера ближнего к линии дислокации), то зависимость деформации от напряжения имеет вид, показанный на рис. 5. Естественно, при надбарьерном движении дислокации зависимость напряжения от деформации имеет упругий характер. При изменении $x$ от 86 до 86.1 (рис. 4) (область спуска, ближнего к линии дислокации барьера) зависимость напряжения от деформации имеет вид, представленный на рис. 6 , - область пластичности.

Таким образом, дислокация на микроуровне периодически переходит из области микропластичности в упругую область.
Математическое моделирование зависимости скорости дислокации от координаты при преодолении барьера Пайерлса дало следующий результат. При движении из долины на потенциальный рельеф скорость дислокации уменьшается, на спуске - растет (потенциальный рельеф - следующая долина). Соответствующий график представлен на рис. 7.

Из рис. 7 видно, что, преодолевая барьер Пайерлса, дислокация действительно движется с переменной скоростью. Такой характер движения дислокаций обусловлен динамическим торможением дислокации, т.е. излучением энергии при преодолении барьера Пайерлса

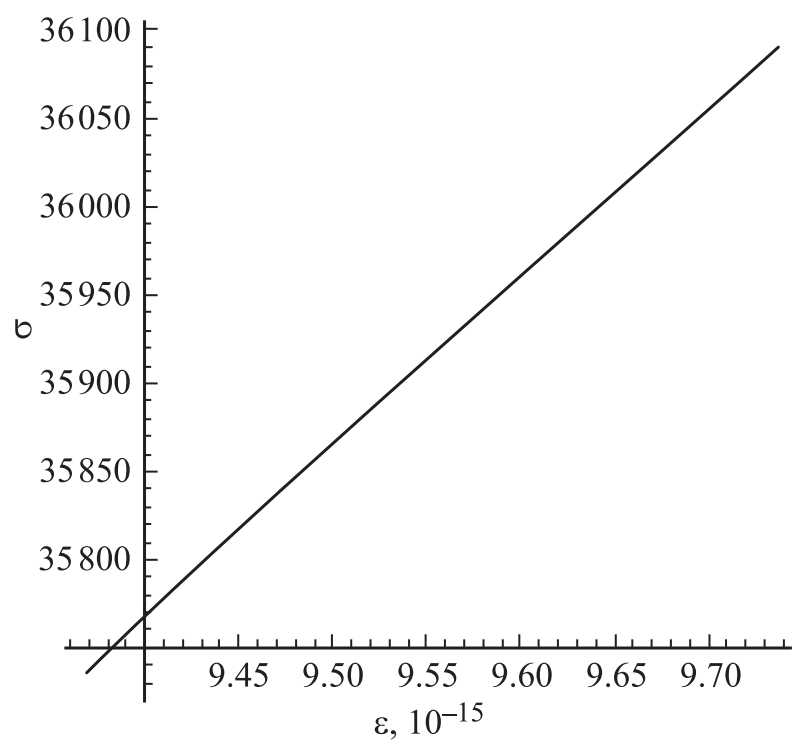

Рис. 5. Зависимость напряжения от деформации при надбарьерном движении.

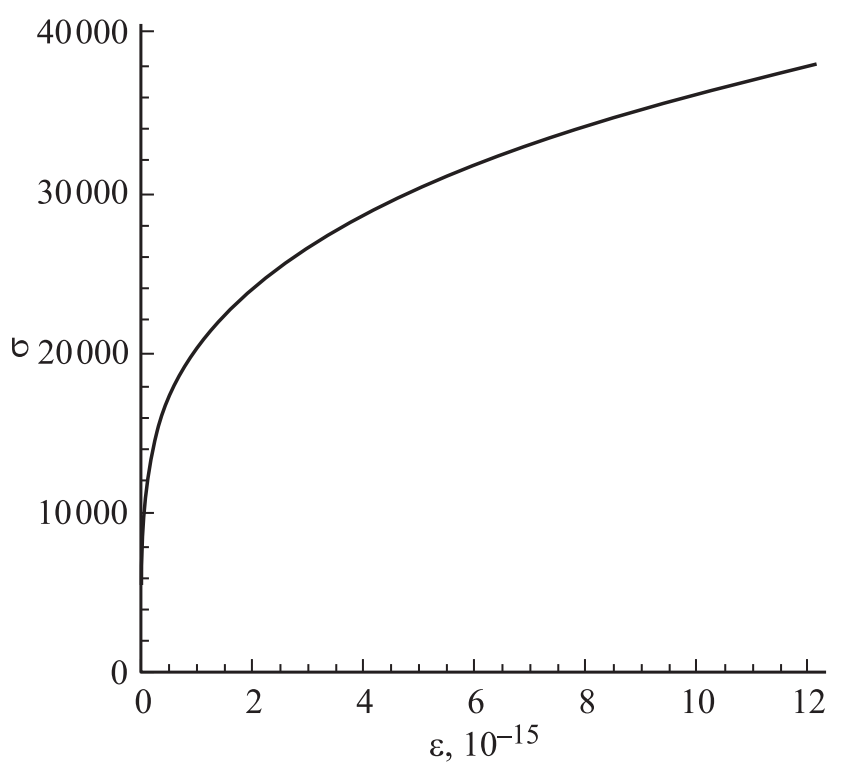

Рис. 6. Зависимость напряжения от деформации для области монотонной зависимости на графике $\sigma(x)$. 


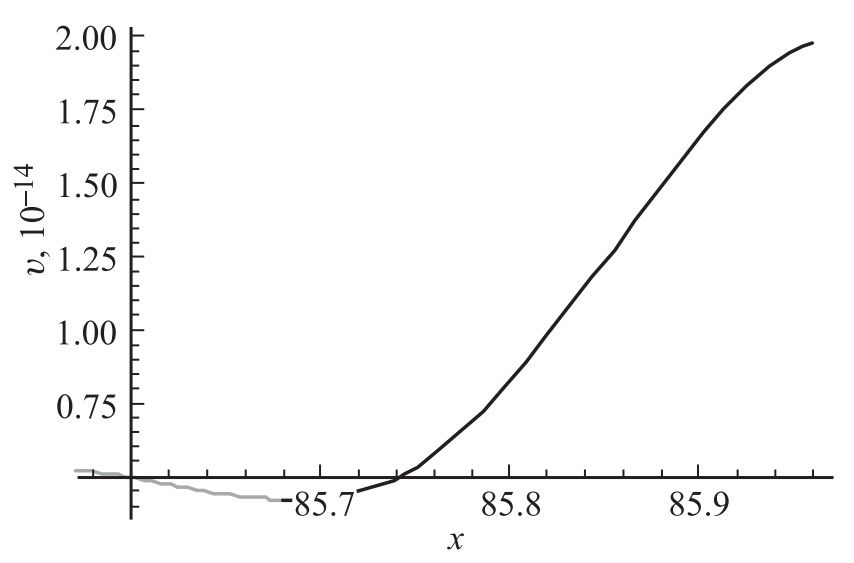

Рис. 7. Зависимость скорости дислокации от координаты в окрестности барьера Пайерлса.

в результате изменения конфигурации ядра дислокации и неравномерности скольжения. При замедлении движения дислокации в макромасштабе получаем деформационное упрочнение. Таким образом, исследование микрохарактеристик при движении дислокации дает результаты, которые объясняют поведение движущихся дислокаций на макроуровне. Предположение, что в окрестности дислокации имеет место микропластичность, оправдано, так как полученные закономерности в макромасштабе дают результаты, соответствующие известным экспериментальным данным.

При низких температурах в алюминии наблюдается аномально высокая скорость ползучести [7]. Покажем, что на макроуровне при этом происходит разупрочнение кристалла. Из синус-уравнения Гордона (2) без правой части получаем функцию перемещений. Далее используем соотношения между деформациями и перемещениями со значениями упругих констант для алюминия и зависимость напряжения от деформации, скорости деформации и температуры при разупрочнении [8]

$$
\begin{gathered}
\sigma=a_{0} \varepsilon^{a_{1}} \xi^{a_{2}} \exp ^{-a_{3} \theta}, \quad a_{0}=3.6 \cdot 10^{6}, \\
a_{1}=0.255, \quad a_{2}=0.05, \quad a_{3}=-0.01,
\end{gathered}
$$

где $a_{0}, a_{1}, a_{2}, a_{3}-$ константы материала, $\xi-$ скорость деформации, $\theta-$ температура. При этом сначала в синус-уравнении Гордона берем высокий барьер Пайерлса, в уравнении (4) - комнатные температуры и $a_{2}=0.05$. Получим зависимость $\sigma(\varepsilon)$, см. рис. 8. Затем берем низкий барьер Пайерлса, низкие температуры, $a_{2}=0.05$ и вновь получим зависимость $\sigma(\varepsilon)$, см. рис. 9. Как видно, ход кривых аналогичен, т.е. действие высокого барьера Пайерлса аналогично действию низких температур или, иначе, с ростом температуры барьер Пайерлса уменьшается, что находится в согласии с [2]. Кроме того, из рис. 8,9 следует, что при $a_{2}=0.05$ имеет место резкое разупрочнение.

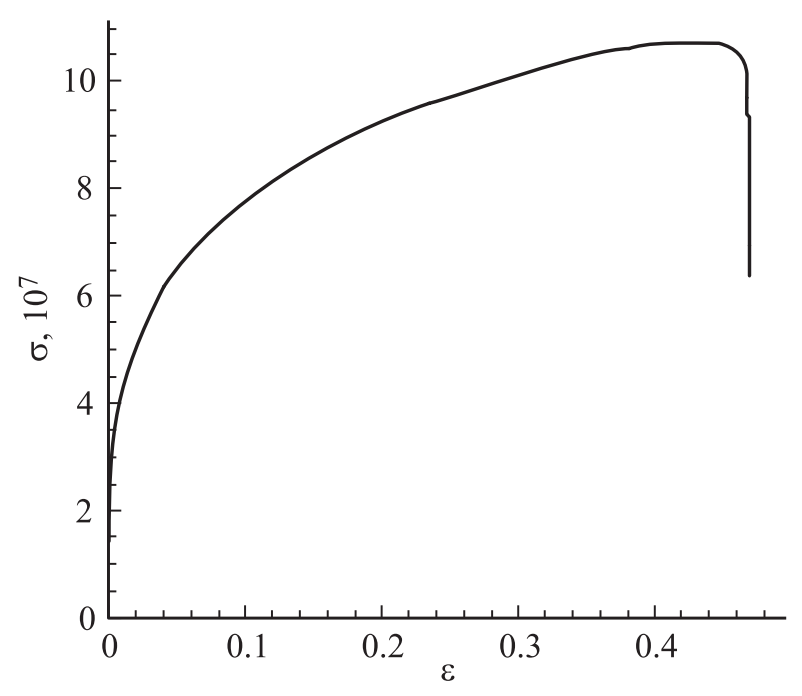

Рис. 8. $\sigma(\varepsilon)$ : высокий барьер, комнатные температуры.

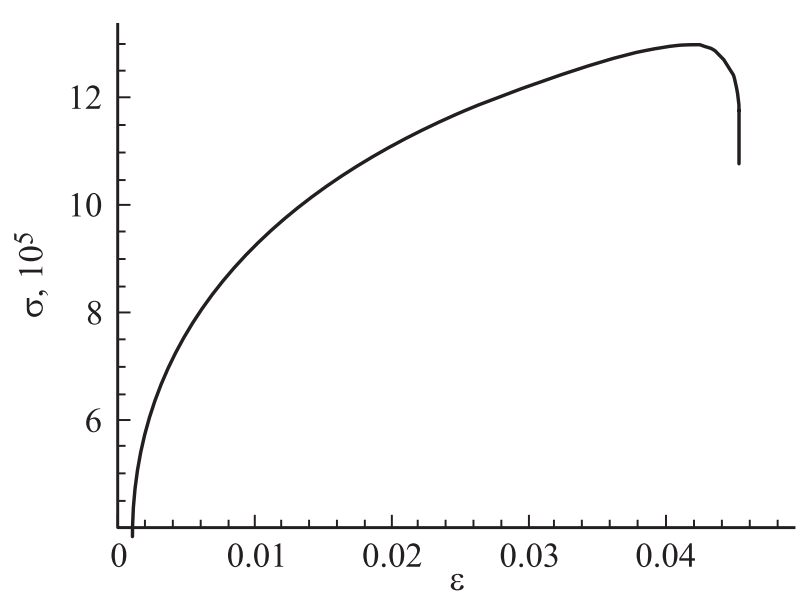

Рис. 9. $\sigma(\varepsilon)$ : низкий барьер, низкие температуры.

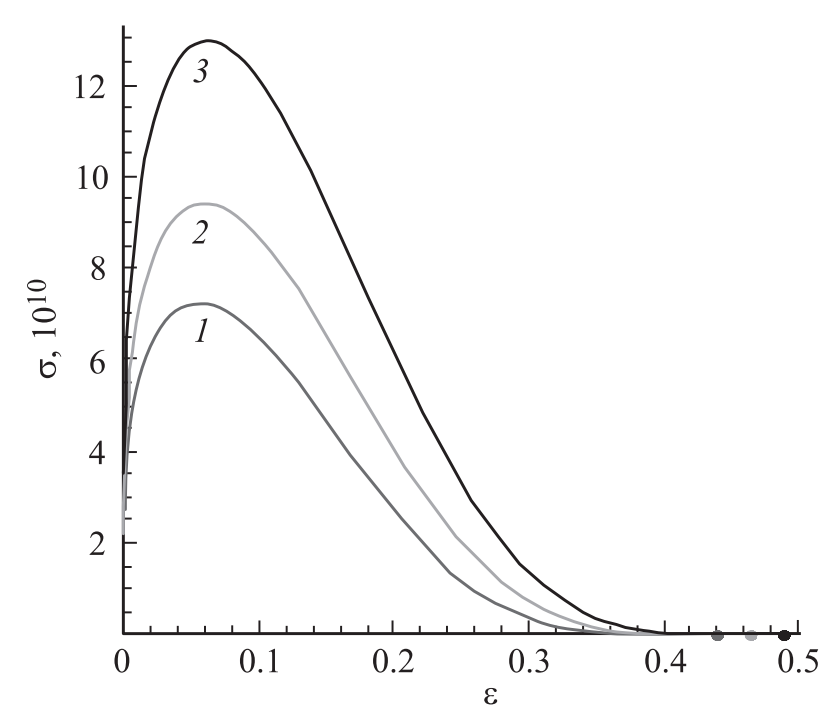

Рис. 10. Зависимость напряжения от деформации для $a_{2}=10$ в присутствии высокочастотного звукового поля. $1-$ резонансная частота $\left(\sim 10^{12} \mathrm{~Hz}\right), 2-\left(\sim 10^{13} \mathrm{~Hz}\right), 3-$ $\left(\sim 10^{11} \mathrm{~Hz}\right)$. 
Для $a_{2}=10$, комнатных температур, высоком барьере Пайерлса и наличии внешнего упругого поля резонансной частоты $\left(\sim 10^{12} \mathrm{~Hz}\right)$ и близлежащих частот $\left(\sim 10^{13} \mathrm{~Hz}, \sim 10^{11} \mathrm{~Hz}\right)$ численный эксперимент дает кривые, представленные на рис 10.

Как видно из рис. 10, при резонансной частоте уменьшается сопротивление деформированию, укорачивается стадия упрочнения и удлиняется стадия разупрочнения.

Таким образом, изучение совместного влияния температуры и скорости деформации на процесс деформации показало, что при низкой температуре и $a_{2}=0.05$ после длительной стадии упрочнения происходит резкое разупрочнение, при высоких температурах, $a_{2}=10$ и наличии внешнего упругого поля наблюдаются плавные стадии упрочнения и разупрочнения, при этом область упрочнения сжимается, а область разупрочнения расширяется, по сравнению с предыдущим случаем.

В ы в о ды.

1. В алюминии при комнатной температуре дислокация в рельефе Пайерлса движется неравномерно, замедляясь перед барьером Пайерлса и ускоряясь после преодоления барьера. В окрестности дислокации реализуется область микропластичности.

2. Внешнее переменное упругое поле изменяет длину свободного пробега дислокации в зависимости от частоты. Барьер Пайерлса существенно меньше и, соответственно, длина свободного пробега дислокации максимальна для резонансной частоты. Закрепление дислокации после прохождения некоторого расстояния говорит об упрочнении.

3. При наличии внешнего упругого поля градиент роста кривой упрочнения уменьшается, а значит уменьшается предел текучести. Различаются 3 области, на которых действуют различные механизмы деформации и упрочнения, каждому участку соответствует различная зависимость коэффициента упрочнения от координаты. Наличие резонансной частоты уменьшает коэффициент упрочнения.

4. Зависимость напряжения от деформации в присутствии высокочастотного звукового поля различна для разных скоростей деформаций, напряжение наименьшее при резонансной частоте.

5. Действие высокого барьера Пайерлса аналогично действию низких температур или, иначе, с ростом температуры барьер Пайерлса уменьшается.

\section{Список литературы}

[1] М.М. Мышляев, В.В. Шпейзман, В.В. Клубович, М.М. Кулак, Г. Лю. ФТТ 57, 1986 (2015).

[2] Д. Хирт, И. Лоте. Теория дислокаций. Атомиздат, М. (1972). $599 \mathrm{c}$.

[3] В.И. Никитенко, Б.Я. Фарбер, Ю.Л. Иунин. Письма в ЖЭТФ 41, 103 (1985).

[4] В.А. Мелик-Шахназаров, И.И. Мирзоева, И.А. Наскидашвили. Письма в ЖЭТФ 43, 247(1986).
[5] W.F. Hosford, R.M. Caddell. Metal Forming. Mechanics and Metallurgy. Cambridge (USA), University Press (2007). 330 p.

[6] А.Н. Орлов Введение в теорию дефектов в кристаллах. Высш. шк. М. (1983). 144 с.

[7] T.A. Parkhomenko, V.V. Pustovalov. Phys. Status. Solidi. A 74, 11 (1982).

[8] Н.Н. Загиров. Теоретические основы пластической деформации. ГАЦМИЗ. Красноярск (1998). 116 с. 